\title{
Role of the integrin-linked kinase/PINCH1/ alpha-parvin complex in cardiac myocyte hypertrophy
}

\author{
Hua Chen ${ }^{1}$, Xueyin N Huang ${ }^{2}$, Wen Yan $^{2}$, Ka Chen ${ }^{2}$, Lida Guo², Lekha Tummalapali², \\ Shoukat Dedhar ${ }^{3}$, René St-Arnaud ${ }^{4}$, Chuanyue $\mathrm{Wu}^{2}$ and Jorge L Sepulveda ${ }^{2}$ \\ ${ }^{1}$ Department of Pathology, New York University, New York, NY, USA; ${ }^{2}$ Department of Pathology, University of \\ Pittsburgh, Pittsburgh, PA, USA; ${ }^{3}$ Department of Biochemistry and Molecular Biology, University of British \\ Columbia, Vancouver, British Columbia, Canada and ${ }^{4}$ Department of Surgery and Department of Human \\ Genetics, McGill University and Genetics Unit, Shriners Hospital for Children, Montréal, Québec, Canada
}

\begin{abstract}
Outside-in signaling from fibronectin (FN) through integrin receptors has been shown to play an important role in promoting cardiac myocyte hypertrophy and synergizes with other hypertrophic stimuli such as the alphaadrenergic agonist phenylephrine (PE) and mechanical strain. The integrin-linked kinase (ILK) is a critical molecule involved in cell adhesion, motility and survival in nonmyocytes such as fibroblasts and epithelial cells. Its role in cardiac myocytes is unclear. In this study, we demonstrate that (1) ILK forms a complex with PINCH1 and alpha-parvin proteins (IPAP1 complex) in neonatal rat ventricular myocytes; (2) localization of IPAP1 complex proteins to costameres in cardiac myocytes is stimulated by FN, PE and synergistically by the combination of FN and PE in an integrin B1-dependent manner; (3) a dominant-negative mutant lacking the PINCH-binding N-terminus of ILK (ILK-C) prevents costamere association of ILK and alpha-parvin, but not PINCH1; (4) FN- and PE-induced hypertrophy, measured by increased protein/DNA ratio, beating frequency and atrial natriuretic peptide expression, is stimulated by low levels of ILK-C but repressed by high ILK-C expression; and (5) overexpression of ILK-C, as well as deletion of the ILK gene in mouse neonatal ventricular myocytes, induces marked apoptosis of cardiac myocytes. These results suggest that the IPAP1 complex plays an important role in mediating integrin-signaling pathways that regulate cardiac myocyte hypertrophy and resistance to apoptosis.
\end{abstract}

Laboratory Investigation (2005) 85, 1342-1356. doi:10.1038/labinvest.3700345; published online 19 September 2005

Keywords: extracellular matrix; fibronectin; integrin; hypertrophy; ventricular remodeling; signal transduction; apoptosis; cardiac myocyte

The extracellular matrix plays an essential role in the proper development of the heart and in the regulation of cardiac function in response to pathophysiologic signals. However, in the hypertrophic heart, marked changes in extracellular matrix composition accompany the development of fibrosis and impairment in contractile function, leading to heart failure. Attachment of cells to the extracellular matrix is mediated by transmembrane receptors, of which the integrins compose a major group. In addition to their role of coupling the extracellular matrix to the intracellular cytoskele-

Correspondence: Dr JL Sepulveda, MD, PhD, Department of Pathology, University of Pittsburgh, CHP MT 5835, 200 Lothrop, Pittsburgh, PA 15213, USA.

E-mail: sepulvedajl@upmc.edu

Received 17 September 2004; revised 19 July 2005; accepted 20 July 2005; published online 19 September 2005 ton, thus providing mechanical support and a structural scaffold for cell shape, integrins act as a liaison between the cell and the extracellular environment, transmitting signals from the extracellular matrix (outside-in signaling) and changing their affinity for the extracellular matrix in response to intracellular signals (inside-out signaling). In cardiac myocytes, integrin-dependent pathways mediate hypertrophic responses to mechanical stimuli associated with cardiac myocyte $\operatorname{strain}^{1,2}$ and are required for stimulation of hypertrophy by phenylephrine (PE) or endothelin. ${ }^{3-5}$ Integrin function is required for proper cardiac development and cardiac myocyte attachment to extracellular matrix, growth and viability. ${ }^{6}$ Integrin-linked kinase (ILK) is an ankyrin repeat-containing serine-threonine protein kinase that binds to the cytoplasmic domain of $\beta 1, \beta 2$ and $\beta 3$ integrin subunits. ${ }^{7}$ ILK forms a tripartite complex (IPAP1 complex) with the LIM 
domain protein PINCH1, and with alpha-parvin (also known as CH-ILKBP or actopaxin, abbreviated as PARVA herein). ${ }^{8-12}$ Assembly of this complex precedes and is required for localization of ILK to the cell/matrix contact sites, focal adhesions and fibrillar adhesions, ${ }^{8}$ as well as for cell shape, motility and survival. ${ }^{13}$ Moreover, ILK kinase activity is involved in IPAP1 complex assembly and is almost exclusively present in IPAP1 complex-associated ILK. ${ }^{14}$ ILK kinase activity is stimulated by growth factors and by attachment of cells to the extracellular matrix, namely to $\mathrm{FN}^{14-16}$ and is localized to the cytoskeletal fraction. ${ }^{14}$ Inhibition of the formation of the IPAP1 complex or of ILK activity in epithelial cells has resulted in decreased cell spreading and motility, ${ }^{8,14,17}$ growth suppression $^{18}$ and apoptosis, ${ }^{19-22}$ and therefore it has been proposed as a potential target for cancer treatment. ${ }^{23}$ Cardiac hypertrophy reproduces some of the molecular features of the neoplastic phenotype in other organs and has been associated with remodeling of the extracellular matrix and increased expression and cytoskeletal association of fibronectin (FN), vitronectin and paxillin. ${ }^{24}$ Interestingly, ILK and IPAP1 complex assembly are required for cytoskeletal association of paxillin in epithelial cells ${ }^{14}$ and a significant increase in ILK mRNA has been observed in a pressure-overload mouse model of cardiac hypertrophy. ${ }^{25}$

Studies from our laboratory ${ }^{26}$ and others ${ }^{27,28}$ have demonstrated that FN induces cardiac myocyte hypertrophy in neonatal rat ventricular myocytes (NRVM). Moreover, FN-induced hypertrophy depended on intact integrin signaling, as evidenced by inhibition with anti- $\beta 1$-integrin antibody, ${ }^{26-28}$ and potentiated hypertrophy induced by the alphaadrenergic agonist PE. ${ }^{29}$ In this study, we examine the hypothesis that $\mathrm{FN}$ and $\mathrm{PE}$ stimulation regulate the expression and localization of ILK, PARVA and PINCH1 proteins in cardiac myocytes where they may play a role in promoting cellular survival and transducing extracellular signals leading to hypertrophy.

\section{Materials and methods}

\section{Isolation and Culture of NRVM}

Ventricular myocytes were isolated from 1- or 2-dayold newborn Sprague-Dawley rats according to an established protocol ${ }^{26,30}$ and in compliance with the 'Guiding Principles for the Care and Use of Animals.' In brief, ventricles were digested with trypsin and DNAse I and nonmyocytes were removed by preplating in uncoated tissue culture dishes for $1 \mathrm{~h}$. Nonattached cardiac myocytes were plated in tissue culture dishes that were precoated with $0.5 \mu \mathrm{g} / \mathrm{cm}^{2}$ of FN (Sigma, St Louis, MO, USA) or with $100 \mu \mathrm{g} / \mathrm{cm}^{2}$ of gelatin for $3-4 \mathrm{~h}$. Cells were usually plated at a density of 500 cells $/ \mathrm{mm}^{2}$ and cultured for $24 \mathrm{~h}$ in MEM containing $5 \%$ bovine calf serum and $0.1 \mathrm{mM}$ bromodeoxyuridine at $37^{\circ} \mathrm{C}$ in $1 \% \mathrm{CO}_{2}$. Using this protocol, a purity of $90-95 \%$ myocytes is routinely achieved. In some experiments, $1 \mu \mathrm{g} / \mathrm{ml}$ of anti-beta-1-integrin (anti-CD29, clone Ha2/5, no azide/no endotoxin, Pharmingen, San Diego, CA, USA) or rabbit IgG were incorporated at all times in the culture medium. At $24 \mathrm{~h}$ after plating, the medium was replaced with MEM containing $10 \mu \mathrm{g} / \mathrm{ml}$ of transferrin, $10 \mu \mathrm{g} / \mathrm{ml}$ of insulin and $1 \mathrm{mg} / \mathrm{ml}$ of low-endotoxin bovine serum albumin (TIBSA). Unless specifically indicated insulin was omitted from the medium after $48 \mathrm{~h}$ of culture and extracts were collected for mRNA or protein analysis after $72 \mathrm{~h}$ of culture.

\section{Isolation and Culture of Neonatal Mouse Ventricular Myocytes}

Ventricular myocytes were isolated from 1- or 2-dayold newborn mice carrying an ILK allele flanked by loxP sites (ILK-fl) ${ }^{31}$ essentially as described above with the following modifications: (1) collagenase was used instead of trypsin, (2) digestions were performed at $37^{\circ} \mathrm{C}$ instead of room temperature and (3) cells were preplated twice for $1 \mathrm{~h}$. Myocytes were plated in FN-coated plates or glass slides in the presence of adenovirus expressing either the Cre recombinase or bGAL at an m.o.i. of 100, in MEM containing $5 \%$ bovine calf serum and $0.1 \mathrm{mM}$ bromodeoxyuridine. After $24 \mathrm{~h}$, the medium was replaced by TIBSA and a fresh aliquot of adenovirus at the same m.o.i. was added for an additional $24 \mathrm{~h}$. This procedure resulted in deletion of the ILK gene and more than $90 \%$ reduction in ILK mRNA expression in myocytes at $48 \mathrm{~h}$ (results not shown). Presumably because of long protein half-life, significant loss of ILK protein expression was only seen after 5-7 days (Figure 9) in the adenovirusCre-treated cells.

\section{Adenoviral Infection and Plasmid Transfection}

Cardiac myocytes were transfected with $300 \mathrm{ng}$ of pEGFP-FLAG-ILK, ${ }^{8}$ GFP-FLAG-PARVA, ${ }^{10}$ pEGFPFLAG-PINCH $1,{ }^{\circ}$ or pEGFP1 (Clontech, Palo Alto, CA, USA) plasmids, mixed with $0.5 \mu \mathrm{l}$ of Lipofectamine 2000 (Invitrogen, Carlsbad, CA, USA), according to the manufacturer's instructions. DNA/ liposome mixtures were incubated in serum-free OPTIMEM (Invitrogen) and mixed with the NRVM preparation just before plating on day 0. Cells were plated into eight-well glass chamber slides (Lab-Tek II, Nalge Nunc, Naperville, IL, USA) coated with gelatin or FN, and cultured as described above. Visualization of GFP localization was performed every day by fluorescence microscopy of live cells, and immunofluorescence analysis was performed at $72 \mathrm{~h}$ of culture as described below. Transfection efficiency as visualized by GFP expression on day 1 was between 10 and $30 \%$. 
Adenoviral vectors expressing a FLAG epitopetagged ILK deletion mutant containing aminoacids 136-452 (ILK-C) ${ }^{18}$ or $\beta$-galactosidase (bGAL) were grown in 293 cells and purified by $\mathrm{CsCl}_{2}$ ultracentrifugation or used as cell lysate supernatants. Results were similar using either of these preparations. Infection was performed at the time of plating of NRVM preparations on day 0. Cardiac myocytes were mixed with adenoviral particles at an m.o.i. of 10 , resulting in infection of more than $95 \%$ of the NRVM, as assessed by coexpression of GFP on day 1. For higher level of expression, adenoviral vectors were used at m.o.i. of 60 .

\section{Immunofluorescence}

Immunofluorescence was performed as previously described. ${ }^{26}$ Briefly, cardiac myocytes were cultured in eight-well slides, fixed in PBS containing $2 \%$ paraformaldeyde, permeabilized with $0.1 \%$ Triton $\mathrm{X}-100$, and stained with primary and secondary antibodies diluted in PBS containing $0.1 \%$ Tween 20 (PBS-T) and 5\% normal donkey serum. Pimary antibodies included rabbit anti-atrial natriuretic peptide (ANP, Peninsula Laboratories, Belmont, CA, USA), mouse clone 65.1 anti-ILK, anti-PARVA antibody 3B5, and anti-sarcomeric actinin antibody (Sigma \#A7811). Secondary antibodies against mouse or rabbit IgG conjugated to Cy2, Cy3 or Cy5 and affinity purified to remove cross-reactivity with IgG from other species were from Jackson ImmunoResearch Laboratories (West Grove, PA, USA). In selected samples, rhodamine-phalloidin (Sigma) was also added at $100 \mathrm{ng} / \mathrm{ml}$ for visualization of the actin cytoskeleton and sarcomere organization.

\section{Protein Extraction, Immunoblotting and Immunoprecipitation}

Total protein extracts were obtained by washing NRVM cultures three times with ice-cold PBS, followed by lysis in sample loading buffer $(1 \%$ SDS, $60 \mathrm{mM}$ Tris $\mathrm{pH} 6.8,10 \%$ glycerol, $140 \mathrm{mM} \beta$ mercaptoethanol). For extraction of insoluble proteins, cells were washed with PBS and scrapped in a hypotonic buffer (20 mM Tris $\mathrm{pH} 7.4,2 \mathrm{mM}$ EDTA, 2 mM EGTA, with phosphatase and protease inhibitors: $100 \mathrm{mM}$ sodium pyrophosphate, $50 \mathrm{mM} \mathrm{NaF}$, $2 \mathrm{mM} \mathrm{Na}_{3} \mathrm{VO}_{4}$ and HALT protease inhibitor cocktail, Pierce, Rockford, IL, USA) and lysed with a Dounce homogenizer. The extracts were centrifuged at $16000 \mathrm{~g}$ for $15 \mathrm{~min}$ and the pellets were extracted with modified RIPA buffer $(50 \mathrm{mM} \mathrm{KCl}, 50 \mathrm{mM}$ HEPES pH 7.8, 1\% NP40, $0.5 \%$ sodium deoxycholate and $0.1 \%$ SDS with protease and phosphatase inhibitors). Samples for immunoblotting were mixed with SDS-loading buffer, boiled for 5 min, briefly microfuged and loaded into a $10 \%$ polyacrylamide gel. Proteins were electroblotted to PVDF membranes (Amersham, Piscataway, NJ, USA) and nonspecific sites were blocked with
PBS-T (PBS with 0.1\% Tween-20) containing 2\% Carnation low-fat milk, for $1 \mathrm{~h}$. The membranes were then briefly washed and incubated in primary antibody diluted in PBS-T with $2 \%$ milk for $1 \mathrm{~h}$. Primary antibodies included anti-ILK clone 65.1 antibody, anti-PARVA clone 3B5, antisarcomeric alpha-actinin (Sigma \#A7811), anti-vinculin (Sigma \#V9131), anti-ILK rabbit polyclonal (Santa Cruz \#H300), anti-GAPDH clone 6C5 (Research Diagnostics, Inc, Flanders, NJ, USA) and anti- $\beta$-actin (Chemicon, Temecula, CA, USA). After three washes in PBS-T for $5 \mathrm{~min}$, the membranes were incubated in HRP-conjugated anti-mouse or anti-rabbit antibody (Santa Cruz Biotechnology, Santa Cruz, CA, USA) for $1 \mathrm{~h}$. After washing three times for $5 \mathrm{~min}$ in PBS-T, protein bands were visualized with ECL-Plus chemiluminescence (Amersham) and exposed to $\mathrm{X}$-ray film.

The PINCH-ILK-PARVA complex was immunoprecipitated from NRVM with monoclonal antiPARVA antibody 1D4 as previously described. ${ }^{10,17}$ Briefly, the cells were lysed with $1 \%$ Triton X-100 buffer, and the extracts were mixed with $500 \mu \mathrm{l}$ of monoclonal anti- $\alpha$-parvin antibody 1D4, precipitated with Protein G (Pierce) and the bound proteins were released from the beads by boiling in SDSloading buffer. The samples were analyzed by Western blotting with anti-PARVA antibody 3B5, monoclonal anti-ILK antibody 65.1 and rabbit polyclonal anti-PINCH1 antibody.

\section{RNA Purification and Real-Time RT-PCR}

RNA isolation, reverse transcription and quantitative PCR using SYBR Green were performed as described. ${ }^{26}$ Primers for ANP were 5'-CCGATAGATCT GCCCTCTTG and 5'-CTCTGGGCTCCAATCCTGT. Primers for GAPDH were 5'-ATGATTCTACCCACGG CAAG and 5'-CTGGAAGATGGTGATGGGTT.

\section{Protein/DNA Measurement}

Myocytes cultured in 24-well plates under various conditions were lysed in $100 \mu \mathrm{l}$ of PBS containing $0.003 \%$ digitonin and $10 \mathrm{ng} / \mathrm{ml}$ of ribonuclease A for $10 \mathrm{~min}$ at RT, followed by addition of $400 \mu \mathrm{l}$ of $8 \mathrm{M}$ urea. For protein assays, a $20 \mu \mathrm{l}$ aliquot was mixed with $200 \mu \mathrm{l}$ of Nano-Orange reagent (Molecular Probes, Eugene, OR, USA) and measured by fluorimetry according to the manufacturer's instructions. Similarly, another $20 \mu \mathrm{l}$ aliquot was mixed with Pico-Green reagent (Molecular Probes) and DNA concentrations measured against a standard curve, according to the manufacturer's instructions.

\section{Caspase Assay}

Cardiac myocytes were cultured in eight-well slides and infected with adenoviral vectors as described above. After $72 \mathrm{~h}$ of culture cells were washed with 
PBS and incubated for $2 \mathrm{~h}$ at $37^{\circ} \mathrm{C}$ in the presence of a sulforhodamine or fluorescein-labeled caspase substrate (Caspa Tag Caspase-3,7 or Pan-Caspase In-Situ Assay Kit, Chemicon). After washing according to the manufacturer's instructions, the percentage of apoptotic cells was measured by fluorescence microscopy. A minimum of 200 cells were counted for each experimental condition.

\section{Statistical Analysis}

Data are presented as means with error bars representing the standard error of the mean. Differences between control and treated cells were evaluated with the two-tailed, unpaired Student's $t$-test.

\section{Results}

\section{A Complex of ILK, PINCH1 and PARVA is Present in Cardiac Myocytes}

Immunoprecipitation with the anti-PARVA 1D4 antibody was performed in lysates from NRVM cultured in the presence of FN. Co-immunoprecipitated proteins were identified by immunoblotting with anti-ILK and anti-PINCH1 antibodies (Figure 1). The results show that a complex of ILK, PINCH1 and PARVA (IPAP1 complex) is present in cardiac myocytes.

\section{Localization of the IPAP1 Complex Proteins in NRVM}

The localization of ILK, PARVA, and PINCH1 was examined by immunofluorescence or by direct visualization of transfected GFP fusion proteins. Staining of F-actin with phalloidin, which highlights the sarcomeres, was performed to identify colocalization with the Z-disks. Dual staining with phalloidin and antisarcomeric alpha-actinin (not shown) showed that areas of more intense staining with phalloidin correspond to the Z-disks, which contain alpha-actinin. In Figure 2, it is apparent that in gelatin-plated NRVM, ILK staining is associated with few Z-disks, and in fact, much of ILK staining does not colocalize with the Z-disks (d-f, arrows). In contrast, in hypertrophic myocytes plated in FN, the majority of ILK staining is associated with the Z-disks ( $j-1$, arrowheads). These results suggest that in FN-induced hypertrophic NRVM, ILK is associated with the costameres, which are areas of apposition of the sarcolema and the Z-disks of the sarcomeres.

The graphs in Figure 2, panels $\mathrm{m}$ and $\mathrm{n}$, illustrate a quantitative evaluation of costamere localization of endogenous ILK and PARVA in cells plated either in gelatin or $\mathrm{FN}$, in the presence and absence of PE. The results show that both FN and PE are able to increase the percentage of cells with costamere localization of ILK and PARVA, and that PE and FN together result in maximal costamere localization of ILK and PARVA. To confirm the results of endogenous ILK and PARVA, and to examine the localization of PINCH1, for which there is no antibody suitable for immunofluorescence, we transfected NRVM with constructs expressing PINCH1, PARVA and ILK fused to GFP. Representative images of transfected NRVM are shown in Figure 3(a-f) and the quantification of the proportion of cells with costamere localization is shown in panels $\mathrm{g}-\mathrm{i}$. In general, the results agree with the effect of FN and PE in localization of endogenous ILK and PARVA and show a similar pattern for PINCH1, suggesting that costamere localization of all three IPAP1 complex proteins is regulated similarly by $\mathrm{FN}$ and PE. Interestingly, cells transfected with GFP-ILK and GFP-PINCH1 (but not with the pEGFP vector) show significant cell death (results not shown), suggesting that fusion of GFP affects formation of multiprotein complexes involved in cell survival. However, the amount of cell death was less in cells treated with both FN and PE, revealing a synergistic effect of this combination in promoting costamere localization of GFP-ILK and to a lesser degree, GFPPINCH1 (Figure 3g and i).

We have previously shown that FN-dependent hypertrophy required binding to $\beta 1$-integrin, because anti-CD29 antibody was able to significantly reduce hypertrophic assembly and ANP expression. ${ }^{26}$ In order to determine if Z-disk colocalization

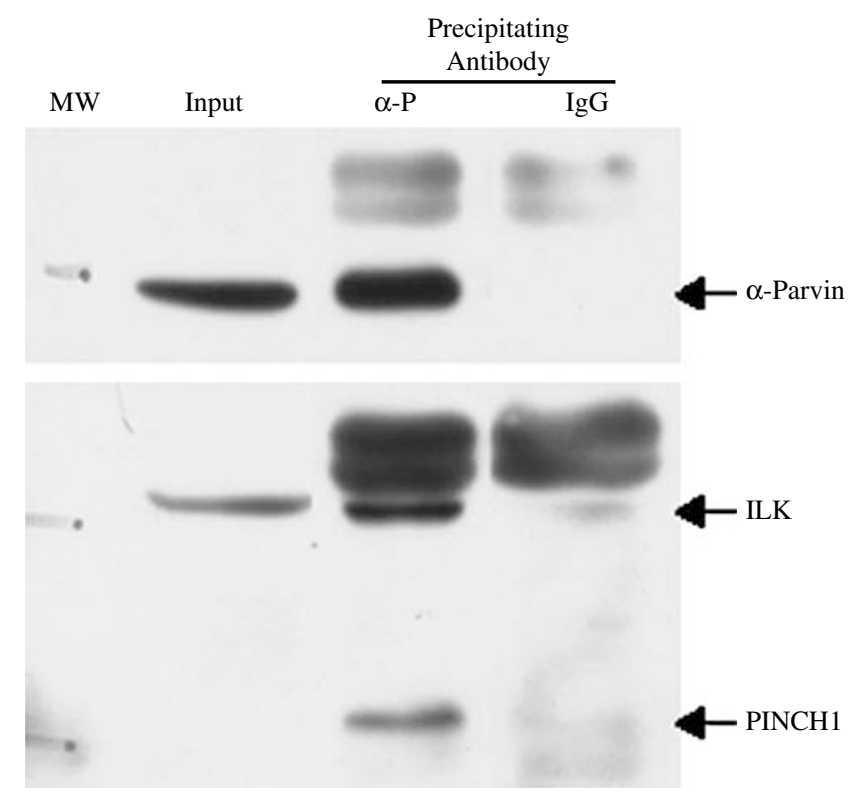

Figure 1 ILK forms a complex with alpha-parvin and PINCH1 in cardiac myocytes. NRVM were plated in FN and extracts collected in $1 \%$ TX-100 buffer after 4 days in culture. Extracts were immunoprecipitated with anti-PARVA antibody 1D4 $(\alpha-P)^{10}$ or with nonimmune IgG (IgG). Immunoprecipitated proteins were immunoblotted with monoclonal anti-PARVA clone 3B5 antibody $^{10}$ (top panel) or with monoclonal anti-ILK clone $65.1^{8}$ and rabbit anti-PINCH1 antibodies ${ }^{17}$ (bottom panel). MW represents molecular weight marker bands in $\mathrm{kDa}$. The input lane visualizes proteins before immunoprecipitation. Note that the PINCH1 band in the input lane is not visible, although it was visible on longer exposure (not shown). The dark bands above the $51 \mathrm{kDa}$ marker are immunoglobulin heavy chains. 
F-Actin
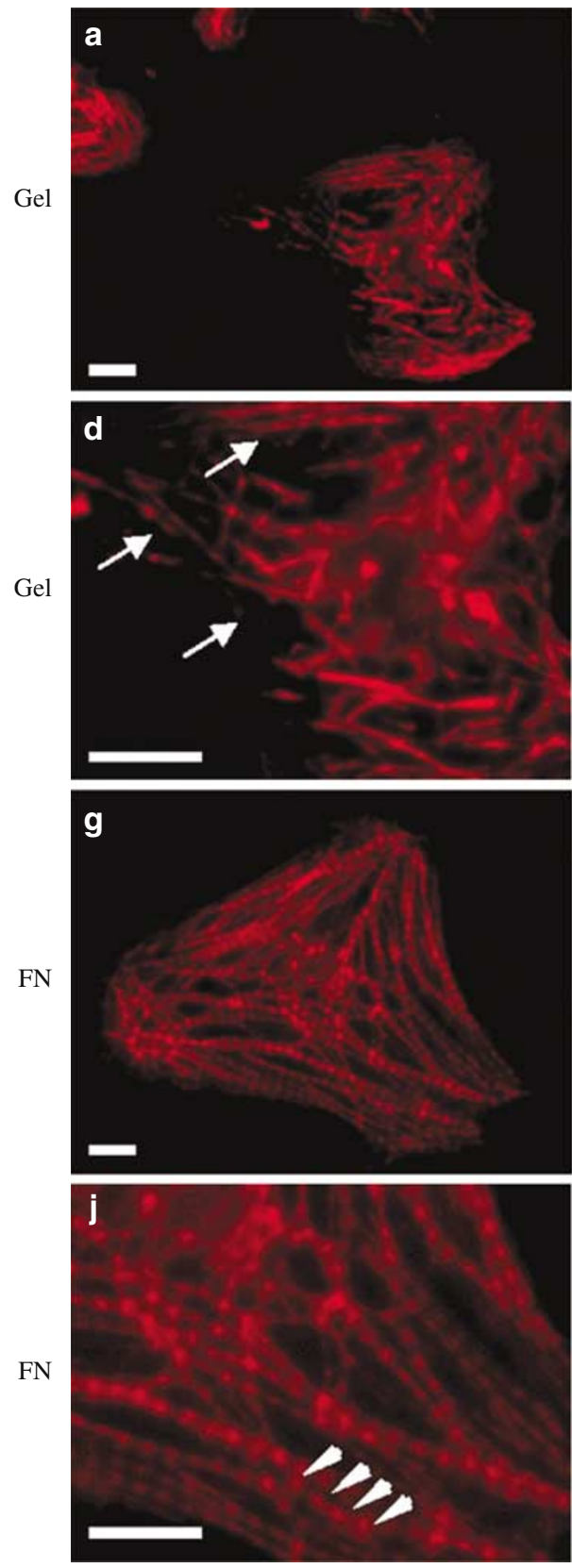

m

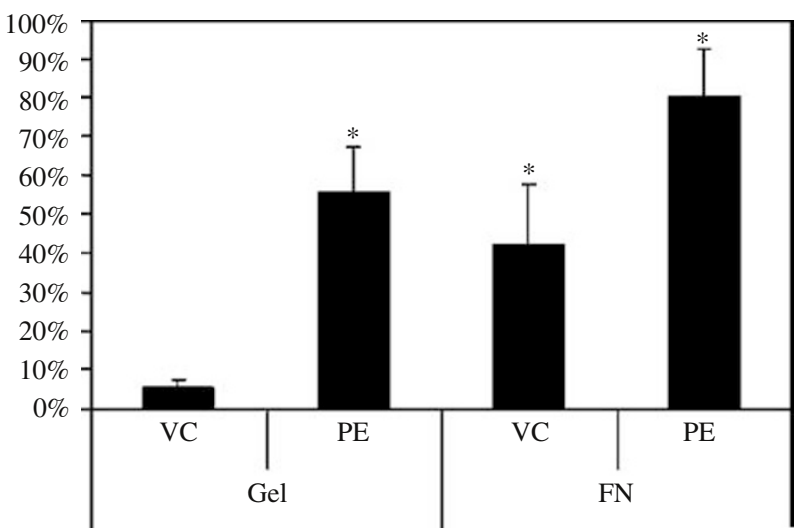

e
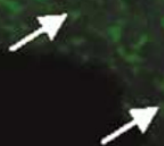

h
ILK
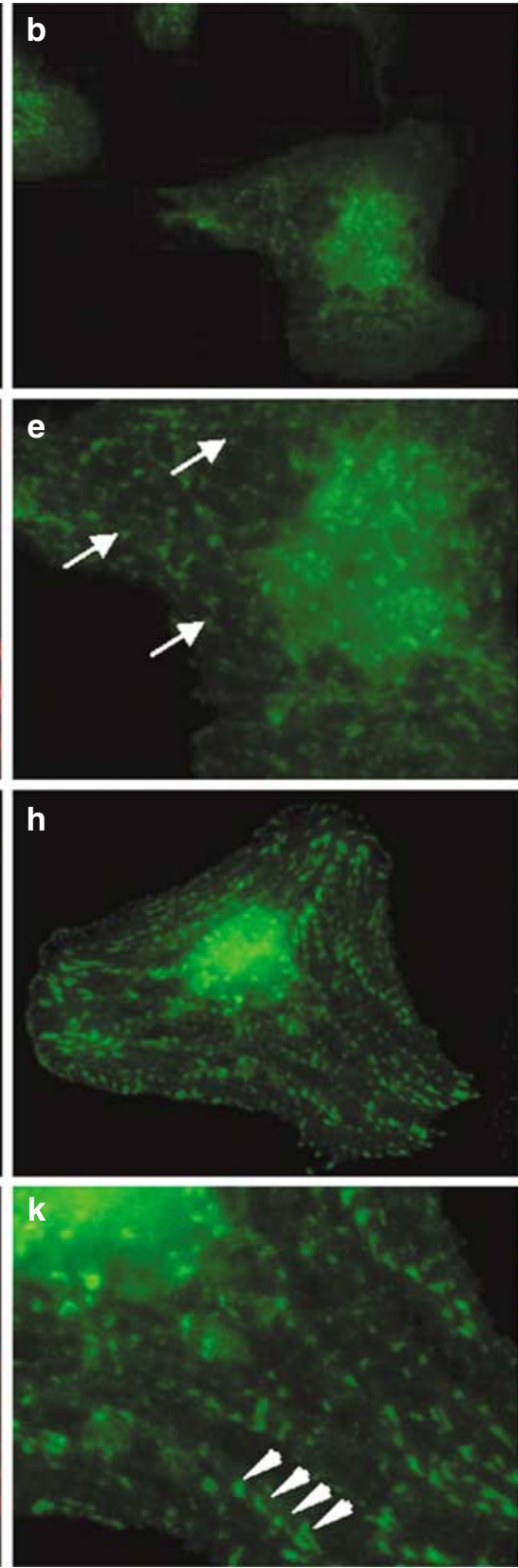

n
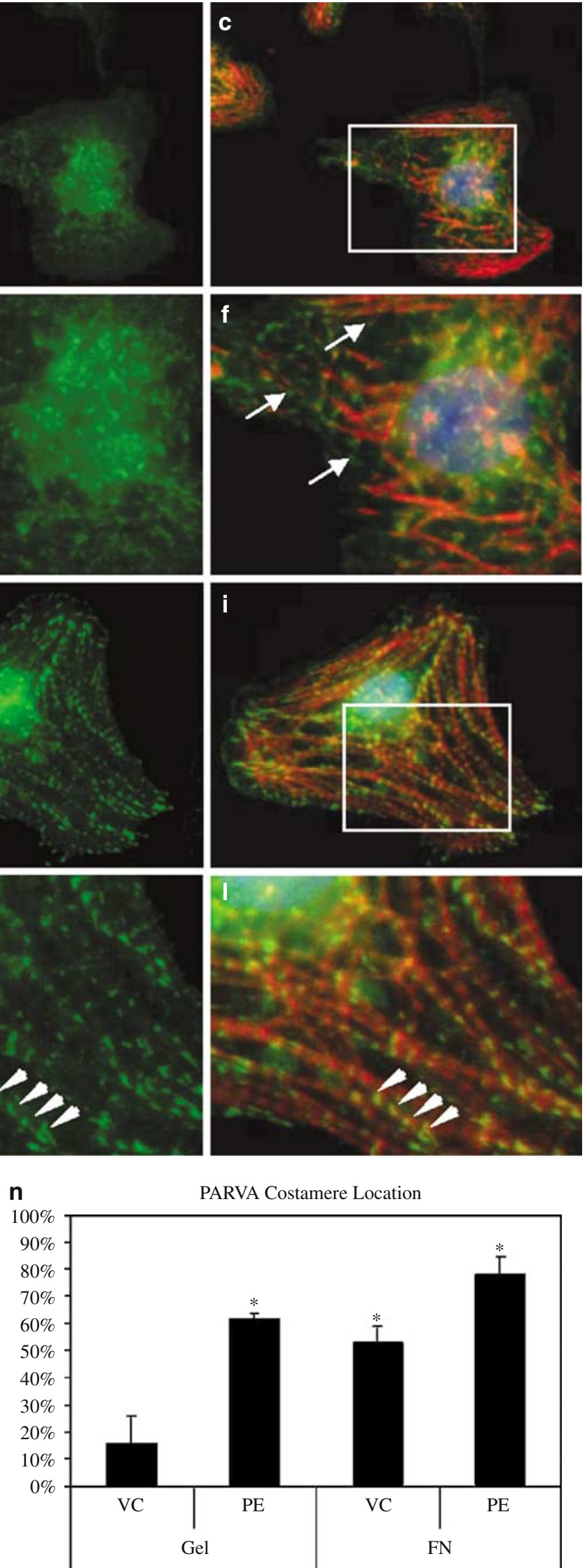

PARVA Costamere Location 
of ILK was dependent on integrin signaling, NRVMs were plated and maintained in the presence of anti$\beta 1$ integrin antibody (anti-CD29) or a similar amount of rabbit IgG. The results show that in addition to inhibiting FN-induced hypertrophy, as shown by a decrease in the expression of ANP (Figure 4, compare a and d), the anti-CD29 antibody also prevented increased Z-disk co-localization of ILK (Figure $4 \mathrm{c}$ and $\mathrm{f}$ ). This result suggests that $\beta 1$ integrin is essential for transmission of the hypertrophic
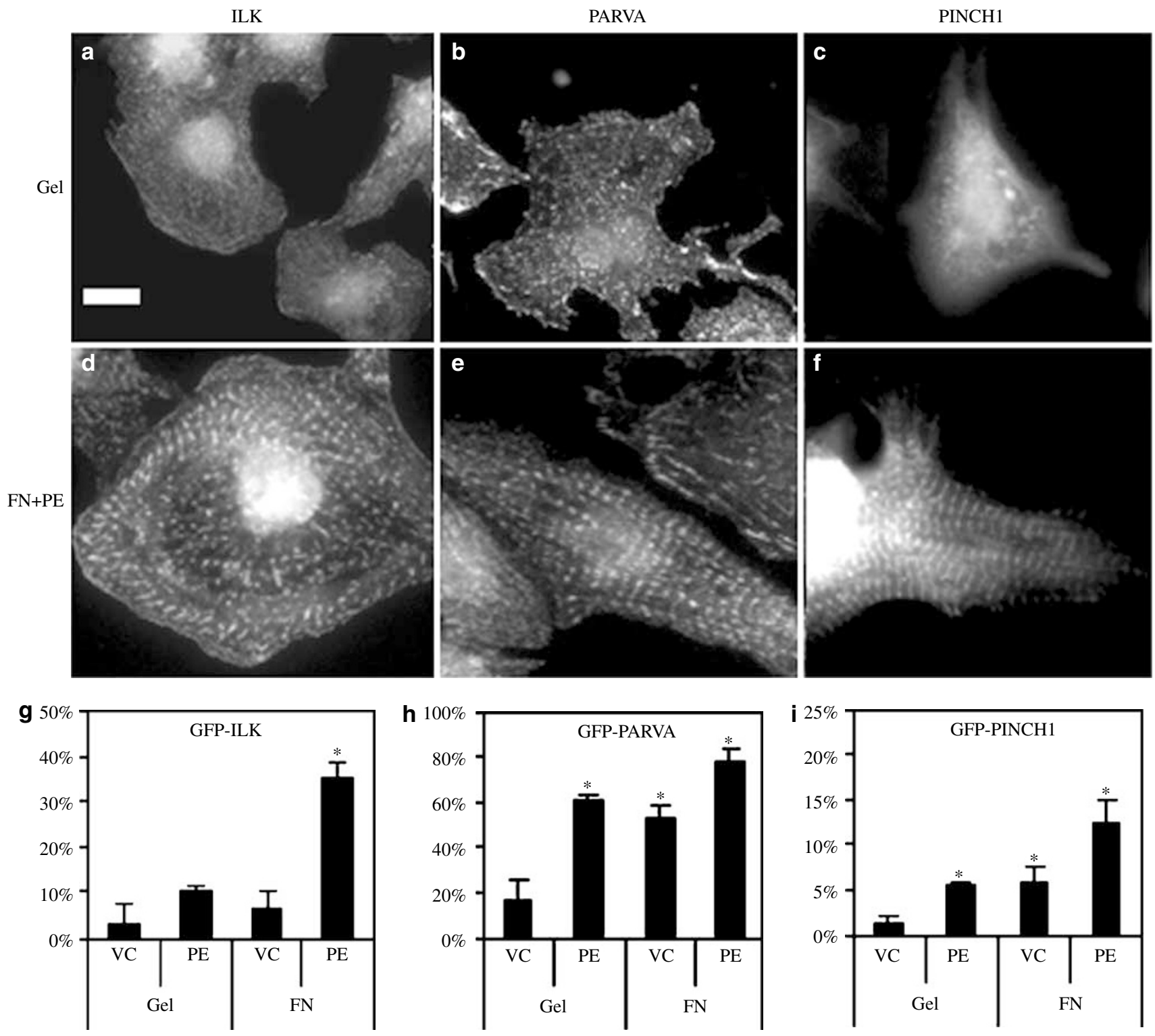

Figure 3 IPAP1-complex proteins colocalization with Z-disks is induced by FN and PE. NRVM were plated in eight-well slides coated with gelatin (a-c) or with FN (d-f) and transfected with GFP-ILK (a, d), GFP-PARVA (b, e) or GFP-PINCH1 (c, f). After $72 \mathrm{~h}$ of culture in the presence of vehicle control (VC, a-c) or phenylephrine (PE, $\mathbf{d}-\mathbf{f}$ ) NRVM were stained with phalloidin-rhodamine (not shown). The white line represents $10 \mu \mathrm{m}$. Quantification of the percentage of NRVM with a costameric localization of GFP-ILK, GFP-PARVA and GFP-PINCH1 fluorescence is shown in panels g-i, respectively. ${ }^{*} P<0.05$ vs control (Gel + VC).

Figure 2 ILK and PARVA colocalization with Z-disks increases in hypertrophic NRVM. NRVM were plated in gelatin (Gel, a-f) or fibronectin-coated eight-well slides (FN, g-l) and cultured for $72 \mathrm{~h}$. After fixation with paraformaldeyde, polymerized actin (F-actin) was visualized with rhodamine-phalloidin (a, d, $\mathbf{g}$ and $\mathbf{j}$ ), and ILK was stained with mouse monoclonal anti-ILK clone 65.1 and Cy2-labeled anti-mouse antibodies ( $\mathbf{b}, \mathbf{e}, \mathbf{h}$ and $\mathbf{k})$. $\mathbf{d}-\mathbf{f}$ represent a high-power view of the field highlighted in (c), and $\mathbf{j}-\mathbf{l}$ represent the field highlighted in (i). Arrowheads point to areas of overlap of ILK with the Z-disks highlighted by the phalloidin staining. Arrows point to ILK staining that does not overlap with Z-disks. The white line represents $10 \mu \mathrm{m}$. Panels $\mathbf{m}$ and $\mathbf{n}$ represent the quantification of the proportion of NRVM with ILK (panel $\mathbf{m}$ ) or PARVA (panel $\mathbf{n}$ ) costamere localization, as measured in at least 200 cells stained with ILK or PARVA antibody and rhodamine-phalloidin in cells plated in gelatin (Gel) or fibronectin (FN) and treated with phenylephrine (PE) or with vehicle control (VC). ${ }^{*} P<0.05$ vs control $(\mathrm{Gel}+\mathrm{VC})$. 
signal from FN, and that both increased costamere localization of ILK and hypertrophic sarcomeric assembly depend on intact integrin signaling.

\section{Effect of a Dominant-Negative Mutant of ILK on IPAP1 Localization}

To interfere with ILK function a deletion mutant of ILK retaining C-terminal amino acids 136-452 (ILK$\mathrm{C}$, which cannot bind PINCH proteins but maintains intact kinase and PARVA-binding domains), with demonstrable dominant-negative activity in other cells, ${ }^{17,18}$ was expressed in NRVM. Infection of NRVM with ILK-C adenovirus resulted in diffuse cytoplasmic expression of ILK-C protein in most cells (Figure 5e) although in several cells a costameric pattern was observed, associated with a hypercontractile phenotype (Figure 5l). Regardless of the pattern of ILK-C expression, NRVM infected with ILK-C adenovirus showed low costameric expression of endogenous ILK (Figure 5f) and PARVA (Figure 5m), while a control bGAL adenovirus had no effect of costameric expression of ILK (Figure 5c) and PARVA (Figure 5i). Interestingly, in the presence of ILK-C, endogenous ILK and PARVA staining in linear pattern corresponding to focal adhesions was maintained or increased in several myocytes (especially in gelatin-plated cells), suggesting that the turnover of these proteins at the focal adhesions is slower compared to the costameres or that ILK-C displaces endogenous ILKPARVA complexes from costameres to focal adhesions. To address the effects of ILK-C on PINCH1 expression, we cotransfected plasmids expressing FLAG-ILK-C and GFP-PINCH1 in NRVM. In Figure 5 , panels $n-p$, we show that ILK-C expression had no effect on costameric localization of PINCH1. These results suggest that ILK-C interferes with ILK and PARVA localization to the costameres, while PINCH1 is still able to localize to costameres.

\section{Association of ILK with the Cytoskeleton}

The results of fluorescence microscopy suggest that the FN hypertrophic stimulus induces costamere localization of IPAP1 complex proteins. In order to validate this conclusion with an independent method, a cytoskeleton-association assay was performed. NRVM plated in gelatin or FN were lysed with a hypotonic buffer and insoluble proteins, including cytoskeletal and nuclear matrix complexes, were separated from soluble proteins and membrane fragments by centrifugation. Proteins associated with the insoluble fraction were solubilized in modified RIPA buffer, separated by SDSPAGE and analyzed by immunoblotting (Figure 6a). In parallel experiments, total protein was obtained from similarly treated NRVM by extraction in $1 \%$
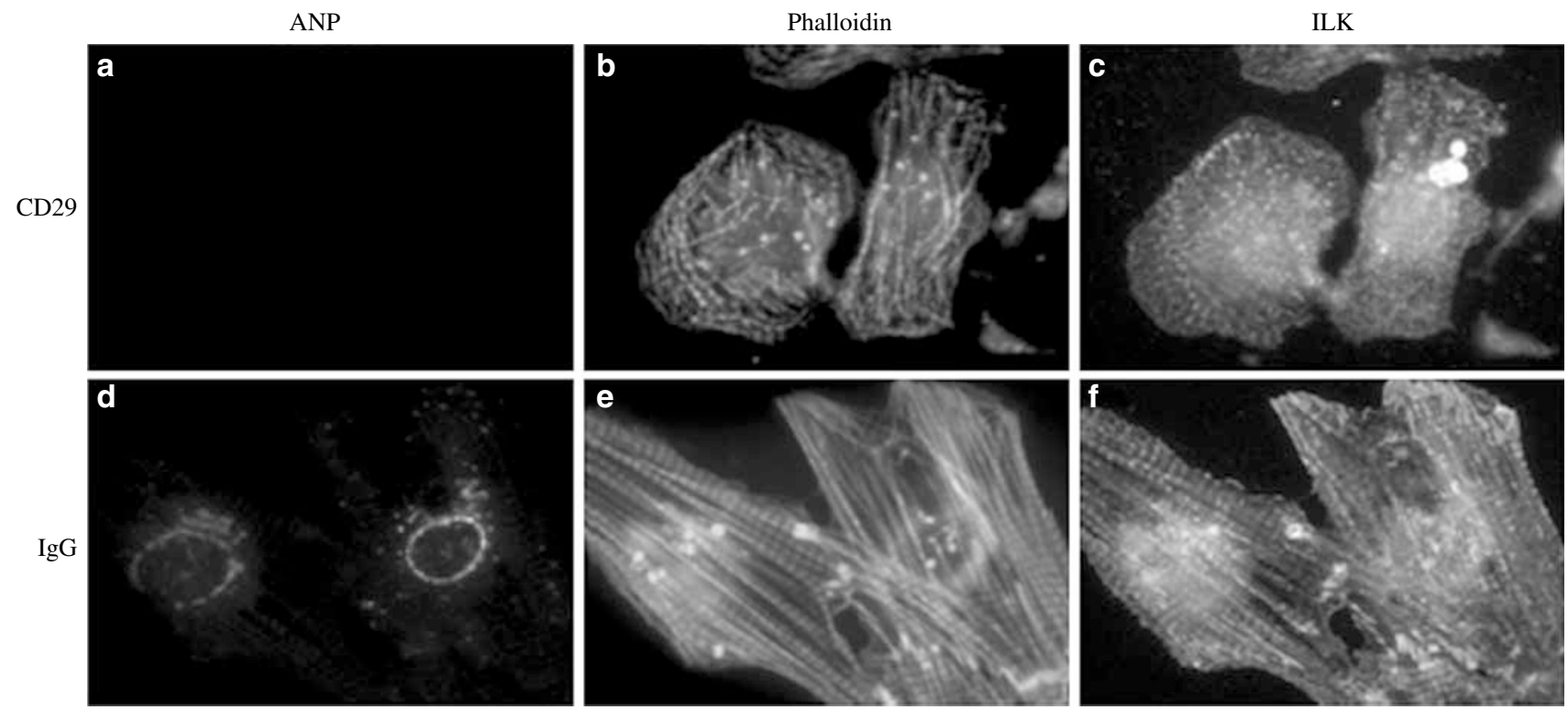

Figure 4 ILK colocalization with Z-disks depends on FN binding to $\beta 1$ integrin. NRVM were plated in FN-coated eight-well slides and cultured in the presence of anti-CD29 (a-c) or nonimmune IgG (d-f). The slides were stained with rabbit anti-ANP and mouse clone 65.1 anti-ILK, followed by Cy5-labeled anti-rabbit and Cy2-labeled anti-mouse antibodies, and rhodamine-phalloidin. a-c and d-f, respectively, represent the same fields viewed with different filters, at the same magnification $(\times 1000)$.

Figure 5 Effect of ILK-C dominant-negative mutant on IPAP1 localization. FN-plated NRVM were infected with adenovirus expressing GFP and ILK-C (d-f, $\mathbf{k}-\mathbf{m})$ or a similar adenovirus that expressed GFP and bGAL $(\mathbf{a}-\mathbf{c}, \mathbf{g}-\mathbf{i})$, or cotransfected with plasmids expressing ILK-C and GFP-PINCH1 (n-p). Expression and localization of ILK-C was determined by immunofluorescence with anti-FLAG antibody, in red $(\mathbf{e}, \mathbf{l}, \mathbf{o})$. Expression and localization of endogenous ILK $(\mathbf{c}, \mathbf{f})$ and PARVA $(\mathbf{i}, \mathbf{m})$ was visualized with specific antibodies that do not react with transfected ILK-C (in yellow). GFP-PINCH1 was visualized by green fluorescence (p). Nuclear morphology was visualized with Hoechst 33242 (a, d, g, k and $\mathbf{n}$ ). The white bar represents $20 \mu \mathrm{m}$. 

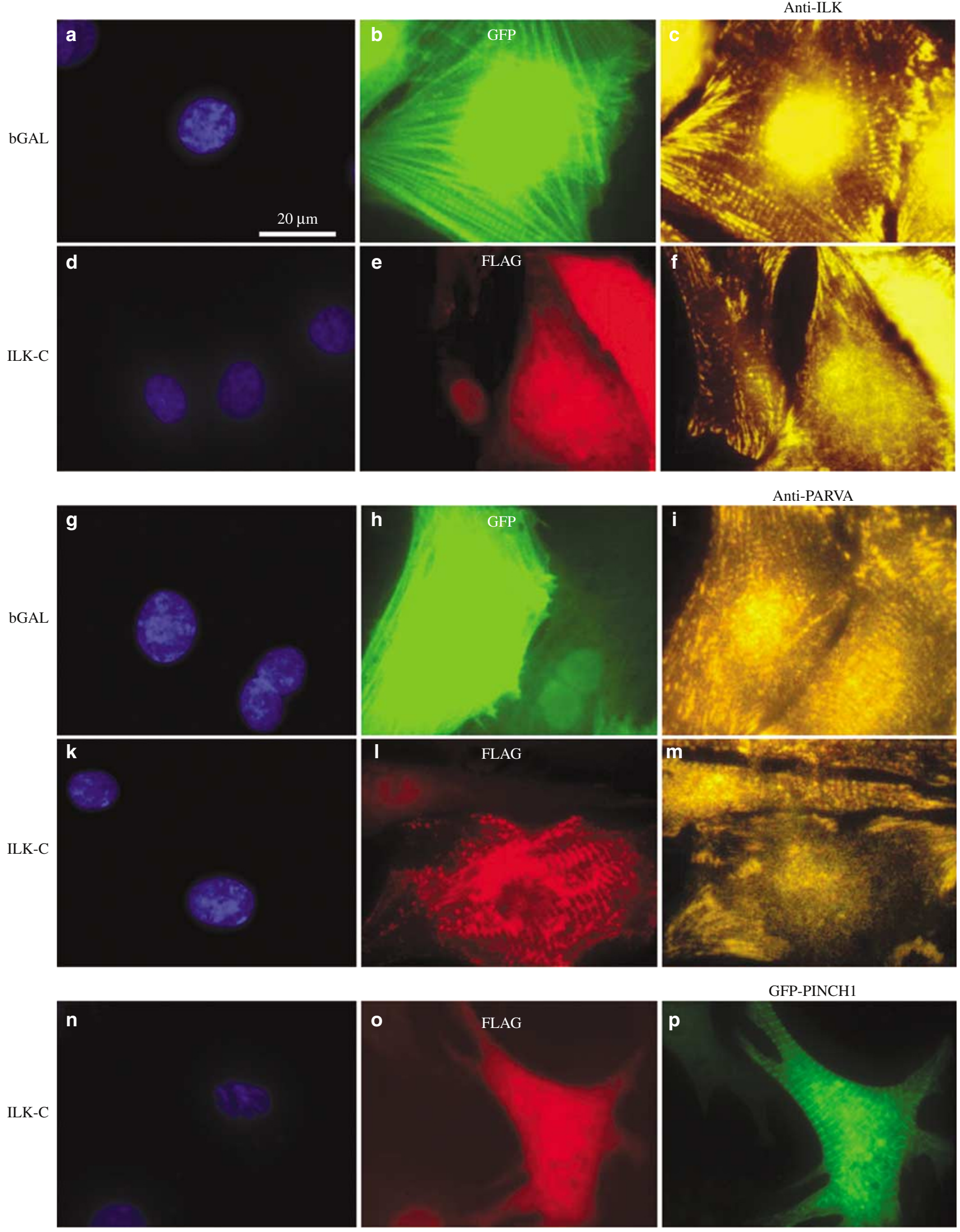

GFP-PINCH1

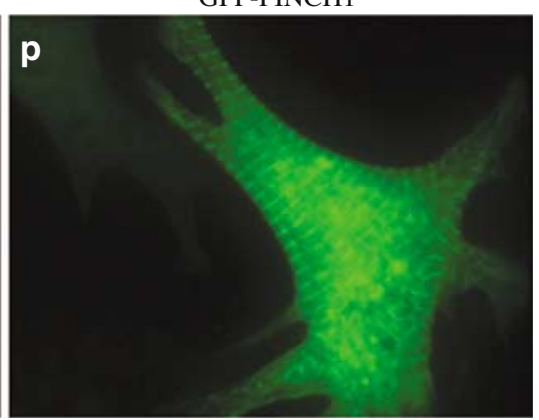


SDS buffer (Figure 6b). The results show that there is little variation in the total amount of ILK or PARVA proteins in the cell with FN treatment, when normalized by $\beta$-actin or GAPDH (Figure 6b). However, FN induced higher levels of ILK and PARVA association with insoluble proteins (Figure 6a, compare lanes 1 and 3). Owing to weak affinity of the antibody, PINCH1 was not detectable in this experiment. Sarcomeric $\alpha$-actinin and vinculin, which are components of the cytoskeleton and costameres, respectively, did not change significantly and can be used for normalization. In the presence of ILK-C, the association of ILK and PARVA with the cytoskeleton was reduced in $\mathrm{FN}$ treated cells (Figure 6a, compare lanes 2 and 4). Sarcomeric $\alpha$-actinin and vinculin were not significantly affected by ILK-C. A mild increase in insoluble ILK and PARVA in gelatin-plated cells treated with ILK-C (Figure 6a, lane 2) might relate to increased localization of ILK and PARVA to focal adhesions, rather than costameres, observed by
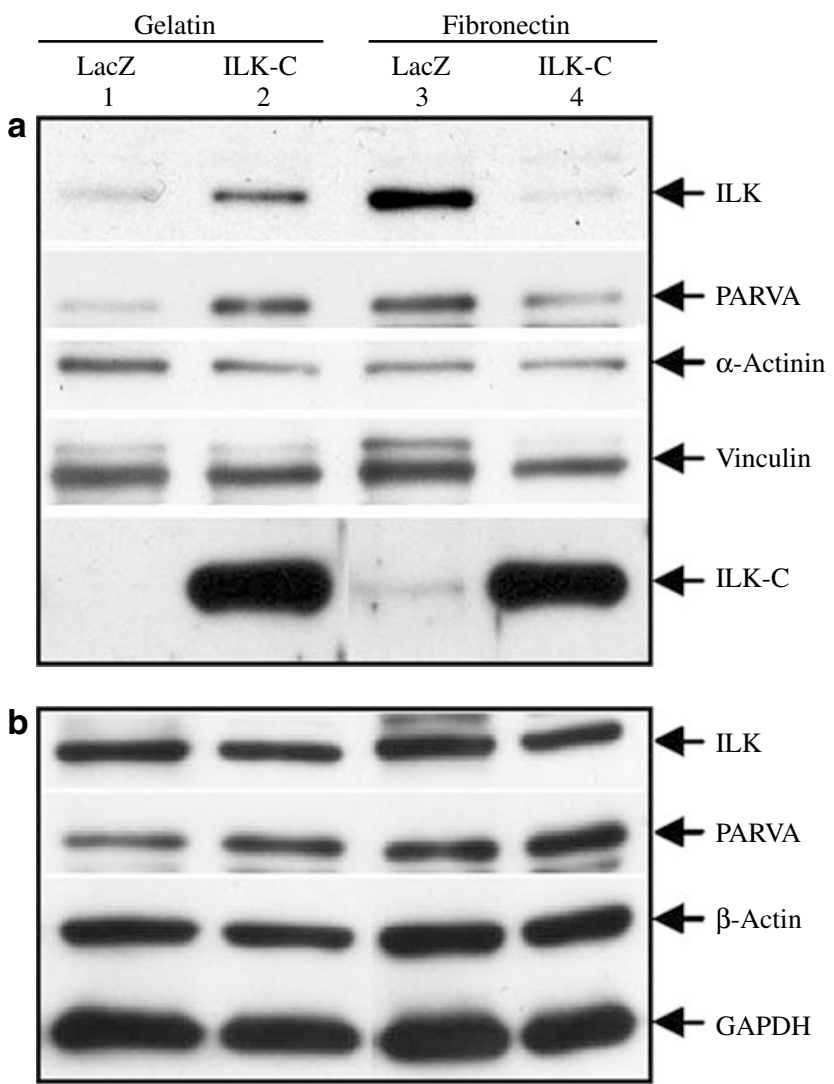

Figure 6 ILK association with insoluble cytoskeletal proteins is increased by FN. NRVM were plated in $10 \mathrm{~cm}$ plates coated with gelatin (Gel) or fibronectin (FN) and treated with bGAL or ILK-C adenovirus at m.o.i of 10 . After $72 \mathrm{~h}$, cells were washed in PBS and lysed in hypotonic buffer. After removal of soluble proteins by centrifugation, the pellets were solubilized in RIPA buffer and separated by immunoblotting (a). For comparison, total protein was extracted from parallel plates in 1\% SDS (b). Bands were successively visualized with antibodies reactive with ILK (N-terminus), PARVA, sarcomeric alpha-actinin, vinculin, the C-terminus of ILK-C, GAPDH and $\beta$-actin, followed by ECL-Plus chemiluminescence (Amersham) immunofluorescence (not shown). Similar results were observed in cells stimulated with $\mathrm{PE}$ or FN plus PE (results not shown). Overall, these results agree with the microscopy observations and together demonstrate that hypertrophic stimulation induces localization of the IPAP1 complex to the costameres and that ILK-C interferes with this process.

\section{Role of ILK in Cardiac Myocyte Hypertrophy}

In order to assess the role of ILK in cardiac myocyte hypertrophy, we investigated the effects of overexpressing the dominant-negative mutant, ILK-C, on the hypertrophic phenotype of NRVM stimulated with FN and/or PE. First we examined the effects of ILK-C on spontaneous beating frequency. FN induced a mild increase in beating frequency compared to gelatinplated cells in bGAL-expressing NRVM (Figure 7a). In contrast, NRVM-expressing ILK-C showed a dosedependent increase in beating frequency, both in FN and gelatin (Figure 7a). bGAL-infected cells treated with $\mathrm{PE}$ showed the maximal beating frequency, which was not further increased by ILK-C and in fact, with high-dose ILK-C it was reduced. In these cells expressing high levels of ILK-C, as surmised by high coexpression of GFP from the adenoviral vector, high beating frequency was often accompanied by a hypercontractile phenotype resulting in small cells with visible sarcomeres by phase contrast microscopy of unstained, live cells (not shown).

Next we examined protein/DNA ratios in gelatin or FN-plated NRVM treated with bGAL or ILK-C adenovirus (Figure 7b). The stimulation of protein synthesis without DNA replication is a feature of hypertrophy and is demonstrated by a 1.8-2.2-fold increase in the protein/DNA ratios of bGAL-treated NRVM stimulated with FN and/or PE. Infection with lower dose of ILK-C adenovirus at 10 m.o.i. was sufficient for induction of protein accumulation in gelatin-plated cells in the absence of PE. However, a higher dose of ILK-C at 60 m.o.i. did not induce higher protein/DNA ratio in gelatin-plated cells and reduced the protein accumulation associated with FN and/or PE stimulation.

ANP expression in NRVM is another hallmark of hypertrophy. We measured average ANP expression by quantitative real-time RT-PCR (Figure 7c) and ANP expression in individual cells by immunofluorescence (Figure $7 \mathrm{~d}-\mathrm{k}$ ). As expected, infection with the control adenovirus did not significantly affect ANP mRNA induction by $\mathrm{FN}$ and/or PE. In contrast, the ILK-C mutant further stimulated ANP expression in FN-plated cells but blocked induction by PE (Figure 7c). In gelatin-plated cells, we observed an increase in ANP expression by immunofluorescence (Figure $7 \mathrm{~d}$ ) but not by RT-PCR (Figure 7c) possibly because of differences in posttranscriptional regulation or because the RT-PCR averages the discrepant expression of different sets of NRVM. When we examined ANP expression in 
cells with high $(++)$ vs low $(+)$ ILK-C expression, it was apparent that marked overexpression of ILK-C significantly blocked ANP induction under all conditions (Figure 8d-g, $\mathrm{j}$ and $\mathrm{k}$ ). This effect was not seen with the control adenovirus (Figure $8 \mathrm{~h}$ and i). Together, these results indicate that mild overexpression of ILK-C results in hypertrophy in the absence of FN or PE and potentiates the hypertrophic effect of FN, while higher levels of ILK-C result in inhibition of the hypertrophic phenotype.

\section{The IPAP1 Complex Plays a Critical Role in Cardiac Myocyte Survival}

Given that transfection of NRVM with GFP-fused IPAP1 proteins increased cell death, as well as published evidence that the IPAP1 complex plays a critical role in promoting cell survival in other cell types, we decided to examine the role of the IPAP1 complex in promoting NRVM survival. First we examined the effects of ILK-C on NRVM survival. Under serum-starved conditions, at $72 \mathrm{~h}$ postplating in gelatin-coated dishes, the proportion of apoptotic cells did not differ between bGAL- and ILK-Cexpressing NRVM (Figure 8a). However, treatment with $\mathrm{PE}, \mathrm{FN}$ or the combination of $\mathrm{PE}$ and $\mathrm{FN}$ significantly reduced the number of apoptotic cells in bGAL-, but not in ILK-C-infected cells (Figure 8), suggesting that ILK-C blocks antiapoptotic signaling mediated by $\mathrm{FN}$ and alpha-adrenergic receptors. When NRVM were treated with $0.5 \mathrm{mM} \mathrm{H}_{2} \mathrm{O}_{2}$ for $2 \mathrm{~h}$, the majority of cells became apoptotic, as assessed by nuclear morphology and caspase activation (Figure 8b-e). Treatment of the NRVM with PE significantly reduced the proportion of apoptotic myocytes. However, expression of the dominantnegative C-terminus of ILK prevented the antiapoptotic effect of PE. With overnight $0.1 \mathrm{mM} \mathrm{H}_{2} \mathrm{O}_{2}$ treatment, only the combination of FN and PE was
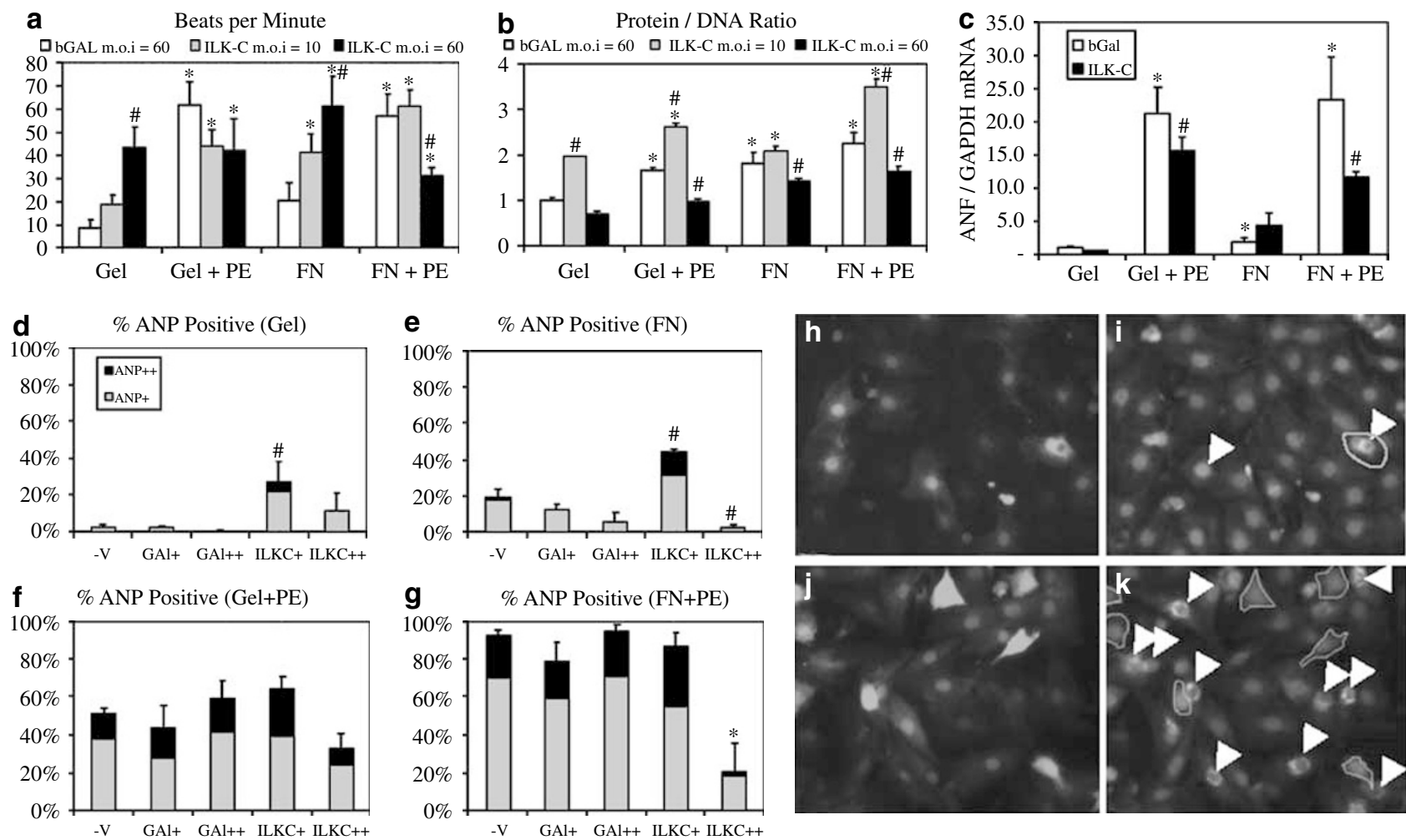

Figure 7 Effect of ILK-C on the hypertrophic phenotype. NRVM were plated in gelatin or fibronectin-coated plates and treated with adenoviral vectors expressing bGAL or the dominant-negative ILK-C mutant at m.o.i of 10 (a-c) or 60 (a-k). Cells were then incubated in the presence of phenylephrine (PE) or vehicle control. * Indicates $P<0.05$ relative to bGAL-treated NRVM plated in gelatin and vehicle control. \# Indicates $P<0.05$ between cells treated with ILK-C and bGAL adenovirus under the same conditions. (a) Beating frequency was measured immediately after removing plates from the incubator after $48 \mathrm{~h}$ of culture. (b) Protein/DNA ratios were measured in triplicate in 24-well plates after $72 \mathrm{~h}$ of culture. (c) RNA was extracted and real-time RT-PCR was performed to quantify ANP and GAPDH mRNAs after $72 \mathrm{~h}$ of culture. The relative ratio of ANP to GAPDH mRNAs is indicated. (d-k) NRVM were plated in gelatin (d, f) or FN-coated (e, g, $\mathbf{h}-\mathbf{k}$ ) eight-well slides and transduced with bGAL (Gal, $\mathbf{d}-\mathbf{g}$, h and $\mathbf{i})$ or ILK-C virus (d-g, $\mathbf{j}$ and $\mathbf{k})$ or untreated with virus (-v, $\mathbf{d}-\mathbf{g})$. After $72 \mathrm{~h}$ of culture in the presence of vehicle control $(\mathbf{d}, \mathbf{e}, \mathbf{h}-\mathbf{k})$ or phenylephrine (f, $\mathbf{g})$, immunofluorescence staining for ANP (i, k) and sarcomeric alpha-actinin (not shown) was performed. GFP was coexpressed from the adenoviral vectors and shown in panels $\mathbf{h}$ and $\mathbf{j}$. d-g: The percentage of NRVM with dim (ANP +) or bright (ANP + +) ANP expression was quantified separately from cells with faint GFP expression (Gal + for bGAL-infected and ILK-C + for ILK-C-infected cells) or bright GFP fluorescence (Gal + + and ILK-C + + ). $\mathbf{h}$ and $\mathbf{i}$ : identical fields showing GFP (h) and ANP (i) expression in bGAL-treated myocytes plated in FN. $\mathbf{j}$ and $\mathbf{k}$ : identical fields showing GFP (h) and ANP (k) expression in ILK-C-treated myocytes plated in FN. The green lines highlight the contours of NRVM expressing high levels of GFP, which show no ANP expression. Arrow heads indicate ANP-positive cells. 
able to synergistically reduce NRVM apoptosis, and this protective effect was also blocked by overexpression of the ILK-C mutant (Figure 8f).
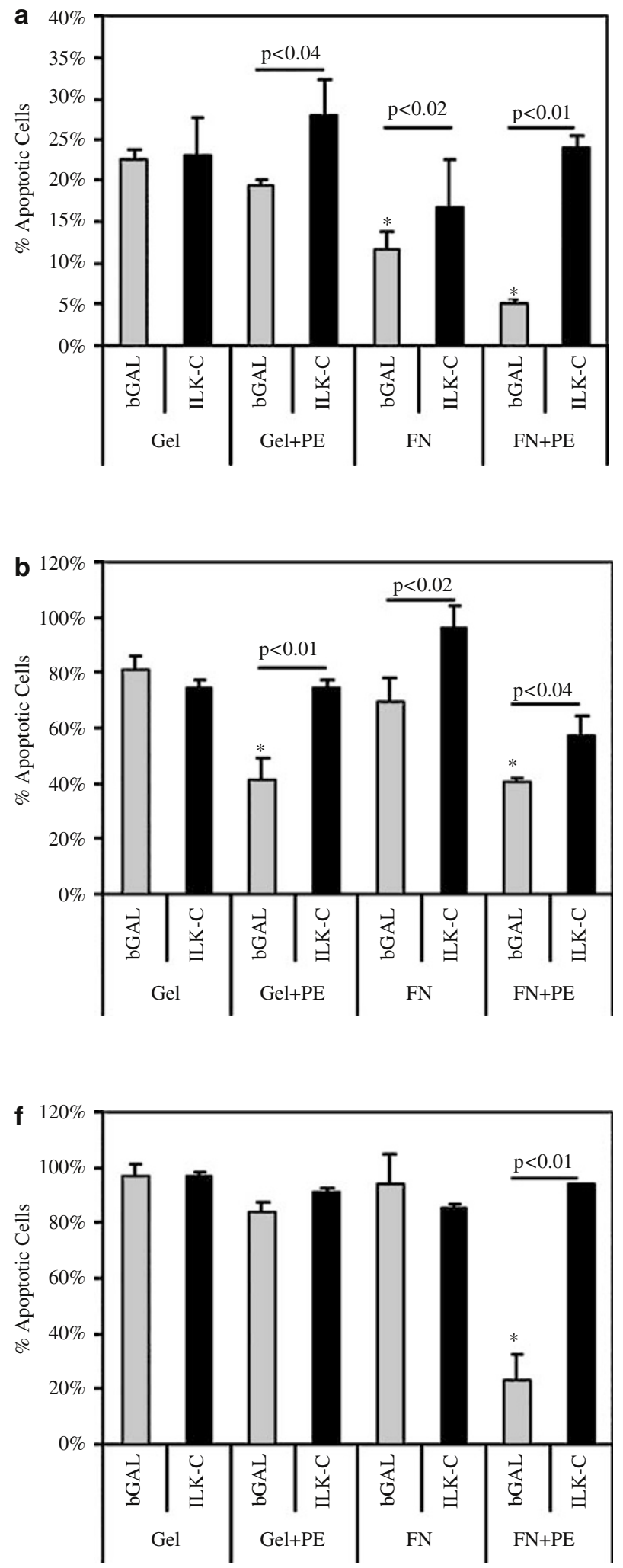

To further demonstrate a role of ILK expression in maintaining cell survival in cardiac myocytes, we deleted the ILK gene in mouse neonatal ventricular

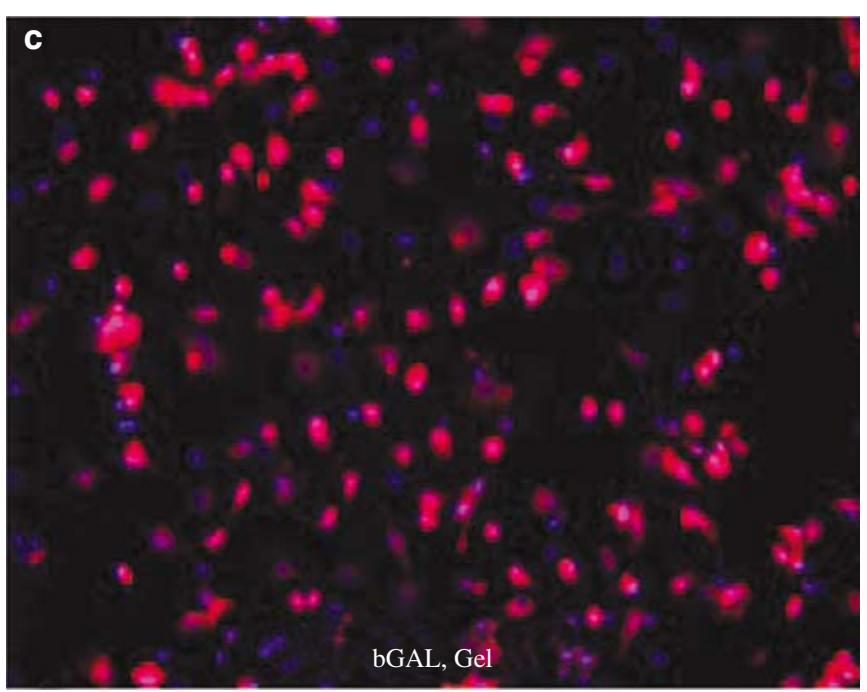

d
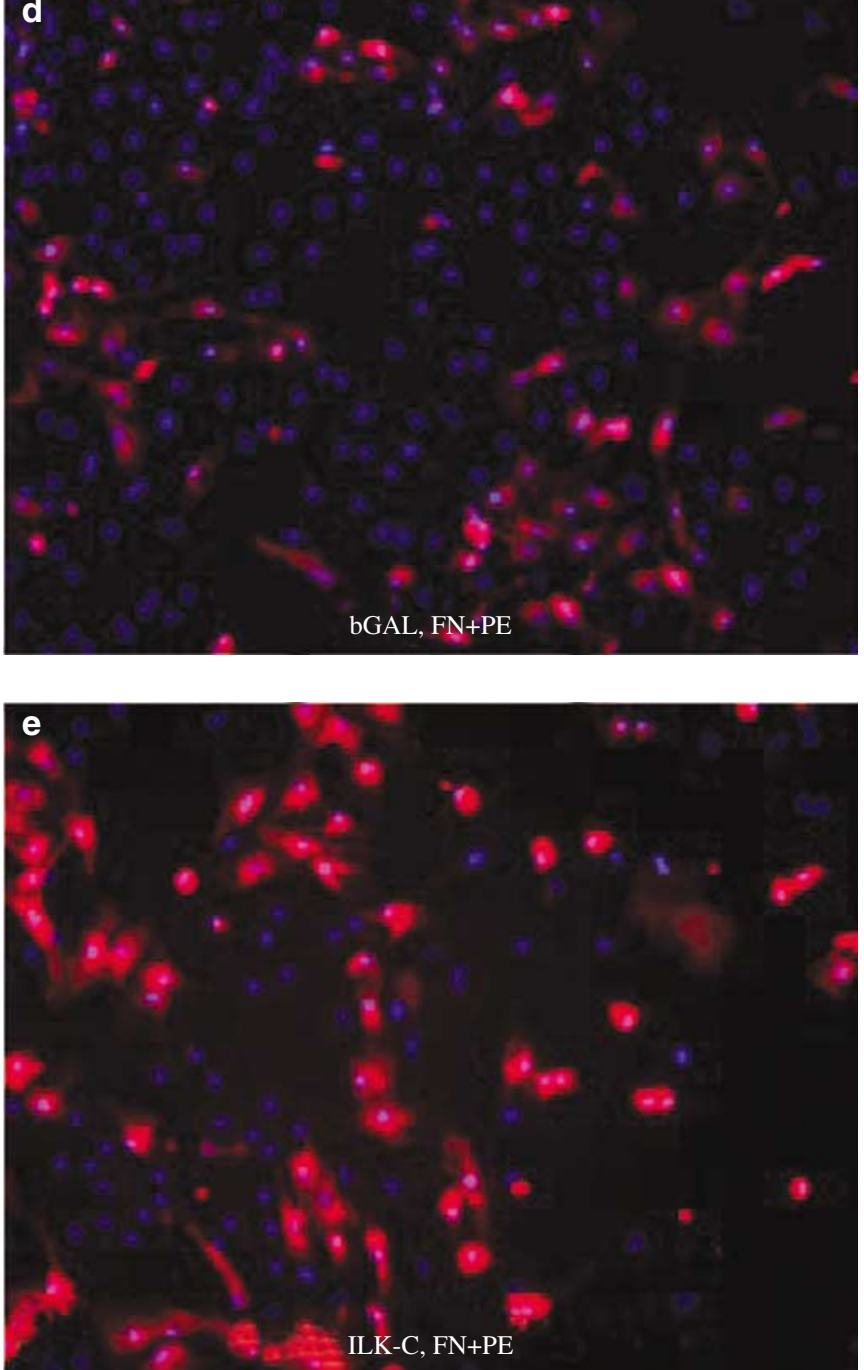
myocytes isolated from mice homozygous for an ILK allele flanked by loxP sites. As demonstrated in Figure 9a, infection with adenovirus expressing Cre recombinase resulted in marked reduction in ILK protein expression after 5-7 days of culture as compared with cells treated with the control lacZ adenovirus. Starting at 6-7 days, an increase in the number of apoptotic myocytes was observed, both by activated caspase staining (Figure 9b-f) and by nuclear morphology (results not shown). ILK deletion resulted in nearly $90 \%$ of cells expressing activated caspases (Figure 9d and f), while the lacZ adenovirus had no effect (c and e). Coinfection with wild-type ILK, but not ILK-C adenovirus, was able to reverse the proapoptotic effect of deleting ILK in mouse myocytes (Figure 9b), confirming that ILK-C is unable to support prosurvival signaling. Together with results of the localization studies, these data support the hypothesis that localization of ILK and
PARVA to costameres is required for the protective effect of integrin and alpha-adrenergic signaling against apoptosis of cardiac myocytes.

\section{Discussion}

In this study, we report for the first time the localization and function of ILK and associated proteins in cardiac myocytes. We demonstrate that ILK forms a complex with PARVA and PINCH1 proteins in NRVM and by two independent methods, immunofluorescence and cytoskeleton association, show that this complex localizes to costameres in a manner regulated by integrin and alphaadrenergic signaling. In both assays, the IPAP1 complex is present in both soluble and sarcomereassociated locations and the total expression of these proteins does not change with hypertrophy. However, coinciding with increased sarcomeric
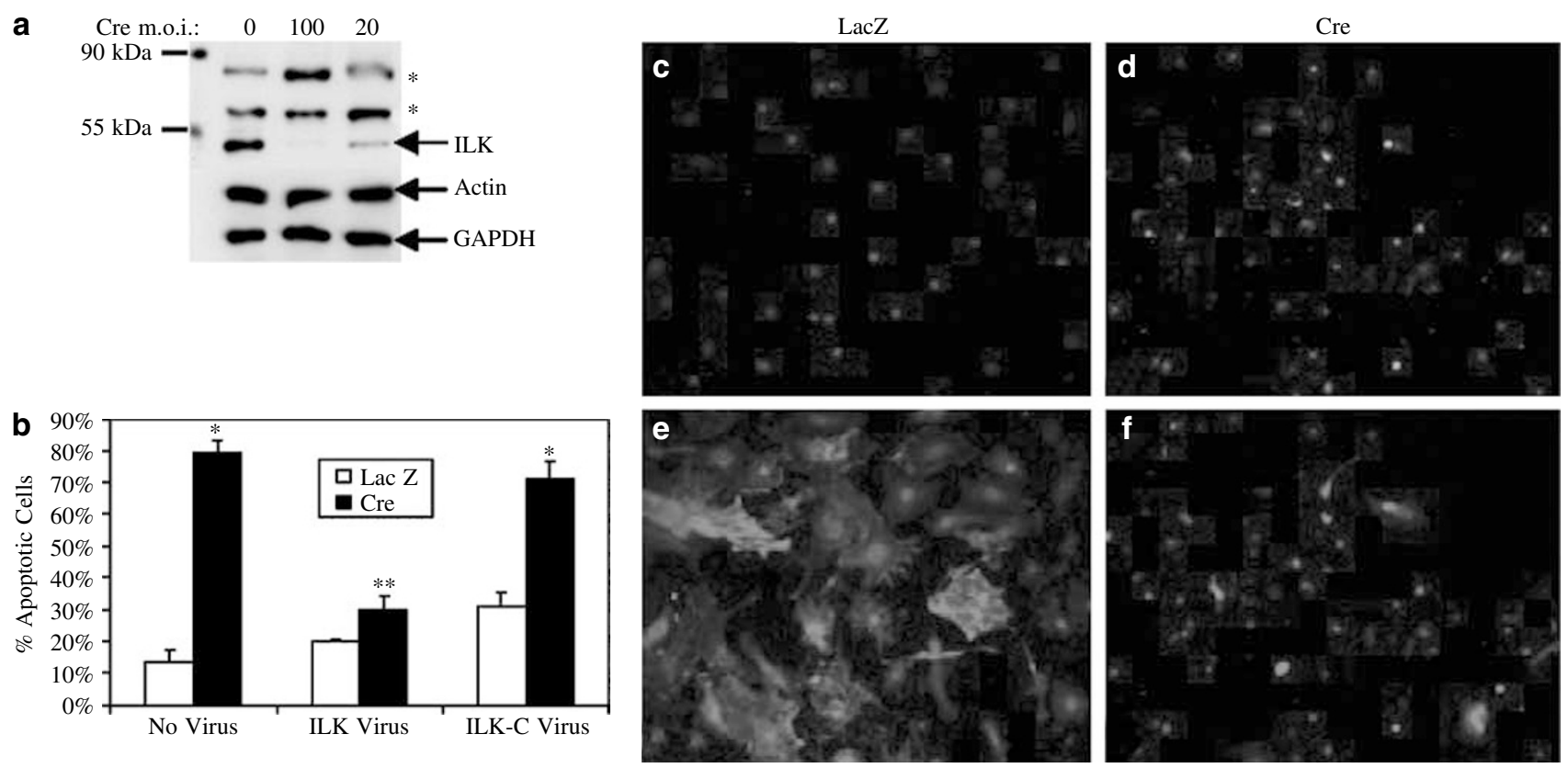

Figure 9 ILK is critical for neonatal mouse ventricular myocyte survival. (a) Mouse cardiac myocytes with floxed ILK gene were treated with adenovirus expressing lacZ (0) or Cre recombinase at 100 m.o.i. Cre adenovirus was also used at 20 m.o.i. Proteins were collected after 7 days and immunoblotted with anti-ILK, $\beta$-actin and GAPDH antibodies. The asterisks indicate nonspecific bands. b-f: Myocytes were treated with 100 m.o.i LacZ (c, e) or Cre (d, f) adenovirus and stained for ILK (red, e, f) or with a fluorescein-linked pan-caspase substrate (green, c, d) . Apoptotic cells show green fluorescence from the FLICA reagent and hyperchromatic, pyknotic nuclei, as assessed by blue fluorescence of the Hoechst 33342 dye (d, f). The graph (b) quantifies the percentage of myocytes with apoptotic nuclei and/or caspase staining. In addition to the Cre or lacZ adenovirus, some plates were coinfected with wild type ILK or ILK-C adenovirus. * Indicates $P<0.001$ between Cre and lacZ virus. ${ }^{*}$ Indicates $P<0.01$ between ILK-C and ILK virus.

Figure 8 ILK-C blocks the antiapoptotic effect of FN and PE. NRVM were plated in gelatin (Gel) or fibronectin (FN) coated eight-well slides and incubated in the presence of vehicle control (Gel, FN) or PE (Gel + PE, FN + PE) for 72 h. Serum was withdrawn at 24 h and the cells were subsequently cultured in MEM with transferrin, insulin and BSA. At plating, NRVM were transduced with adenoviral vectors expressing GFP and either bGAL or the ILK-C dominant-negative mutant. Apoptotic cells were identified after staining of activated caspases with a cell-permeable sulforhodamine-linked caspase-3,7 substrate and the percentage of apoptotic cells is shown in the graph. Apoptotic cells show red fluorescence from the FLICA reagent and hyperchromatic, pyknotic nuclei, as assessed by blue fluorescence of the Hoechst 33342 dye. The percentage of apoptotic cells under the various conditions is shown in graphs a, b and $\mathbf{f}$. Panel a shows the apoptotic response to serum starvation. In panels b-e, $0.5 \mathrm{mM} \mathrm{H}_{2} \mathrm{O}_{2}$ was added for $2 \mathrm{~h}$ and in panel $\mathbf{f}, 0.1 \mathrm{mM} \mathrm{H} \mathrm{H}_{2} \mathrm{O}_{2}$ was added for $18 \mathrm{~h}$. A representative field of pan-caspase staining of NRVM treated with $0.5 \mathrm{mM} \mathrm{H}_{2} \mathrm{O}_{2}$ for $2 \mathrm{~h}$ is shown for $\beta$-gal transduced cells plated in gelatin (c) or in FN + PE (d), and ILK-C-transduced cells plated in FN + PE are shown in (e). Asterisks indicate statistically significant $(P<0.05)$ decreases in the percentage of apoptotic cells in $\beta$-gal-transduced NRVM compared to baseline (Gel). Also indicated are $P$ values for the significant differences between ILK-C- and $\beta$-gal-transduced cells for each condition. 
assembly, there is a shift in IPAP1 protein localization from diffuse to costameric in hypertrophic myocytes. These results agree with previous observations that Z-line sarcomeric distribution of $\mathrm{FN}$, collagen and integrins beta(1A) and alpha1, 3A and 5 increased in hypertrophic cardiac myocytes exposed to PE. ${ }^{32,33}$ Our data do not distinguish between increased recruitment of IPAP1 proteins to newly formed sarcomeres or increased sarcomere nucleation in precostameres enriched in IPAP1 proteins during hypertrophy.

Interestingly, Western blot analysis of total ILK (Figure 6), as well as quantification of ILK mRNA by RT-PCR (not shown), did not demonstrate any significant increase in total ILK mRNA or protein abundance with FN- or PE-induced hypertrophy. These results appear different from those reported in mice, which showed marked induction of ILK mRNA in cardiac hypertrophy caused by aortic banding but not by calcineurin overexpression. ${ }^{25}$ It is possible that differences in the hypertrophic stimuli account for the discrepancies, although we also did not see induction of total ILK protein by mechanical strain in cultured NRVM (results not shown). More likely, induction of ILK expression may occur in nonmyocyte cells subjected to mechanical strain in the aortic banding model.

Integrin signaling has been reported to be essential for cardiac myocyte hypertrophy induced by G-protein-coupled receptors ${ }^{3,4}$ and a synergistic effect of FN and PE in promoting hypertrophy has been previously observed. ${ }^{5,28}$ This effect is partially explained by PE-induced rapid tyrosine phosphorylation of costamere complex proteins, including focal adhesion kinase (FAK), paxillin and p130 Crk-associated substrate, ${ }^{28}$ an effect dependent on intact integrin and FAK functions. Our data demonstrate that $\mathrm{PE}$ also synergizes with $\mathrm{FN}$ in inducing increased costamere localization of ILK and PIPcomplex proteins, suggesting that these proteins play a role in hypertrophic signaling.

Cardiac myocyte hypertrophy is usually characterized by (a) increased global mRNA and protein synthesis resulting in increased sarcomeric assembly and expanded cell area, (b) re-expression of fetal genes, such as ANP, and (c) increased cell survival. We observed all of these features in response to FN induction and demonstrated their dependence on $\mathrm{FN}$ binding to $\beta 1$-integrin (Figure $4^{26}$ ). Since FN and $\mathrm{PE}$ increased localization of IPAP1-complex protein to costameres, it is reasonable to consider that IPAP1 proteins may play a role in any of the phenotypic features of hypertrophy. Indeed we demonstrated that expression of a dominant-negative mutant form of ILK which disrupted costamere localization of endogenous ILK and PARVA prevented the protective effect of FN and PE against apoptosis induced by serum starvation. These results suggest that FN and PE require costamere localization of ILK and PARVA to promote NRVM survival. This hypothesis agrees with observations in fibroblasts and HeLa cells that the assembly of the IPAP1 complex was critical for cell survival, an effect in part mediated by activation of the Akt kinase. ${ }^{9,13}$ In these nonmyocyte cells, assembly, stability and localization of the IPAP1 complex to focal adhesions is dependent on adequate levels of ILK, PINCH1 and PARVA. ${ }^{8,9}$ This protective role of IPAP1 complex concurs with previous observations that ILK-dependent induction of Akt phosphorylation at serine 473 is a critical step of antiapoptotic signaling involving Ras in cardiac myocytes. $^{34}$ While endogenous PARVA and ILK localization to costameres was prevented by ILK-C, transfected GFP-PINCH1 still localized to costameres in the presence of ILK-C. This result may be explained by possible interaction of PINCH1 with other LIM-domain containing proteins or the adaptor Nck2. ${ }^{16}$ In contrast with epithelial cells, where ILK-C cannot locate to focal adhesions, some myocytes expressing high levels of ILK-C showed localization of ILK-C to costameres. ILK-C might localize to costameres because of its ability to bind directly to beta-parvin, ${ }^{10,11,35}$ paxillin, ${ }^{11,14}$ or $\beta$-integrins, ${ }^{7}$ although we must also consider costamere localization a consequence of artificially high levels of ILK-C expression. Cells with high level of ILK-C expression and costamere localization often showed a hypercontractile phenotype with increased beating frequency and rapid progression to apoptosis. Since in these cells endogenous PARVA and ILK were absent from the costameres, it is reasonable to speculate that expression of the intact IPAP1 complex at the costameres is essential for promoting cell survival and perhaps moderating contractility of the sarcomere.

In addition to the effect on cell survival, ILK plays a role in cardiac myocyte hypertrophy. Moderate levels of ILK-C expression induced three different aspects of the hypertrophic phenotype (increased beating frequency, protein accumulation and ANP expression) in the absence of FN or PE. Since PARVA has been demonstrated to repress Rac1 activity in other cell types, ${ }^{36}$ we hypothesize that in the presence of ILK-C, decreased costameric PARVA causes activation of Rac1, resulting in increased beating, sarcomere assembly and hypertrophy. Alternatively, expression of ILK-C may interfere with the balance of alpha- and beta-parvin interactions with ILK and affect Rac1 expression, since PARVA represses and beta-parvin enhances Rac1 activity. ${ }^{36}$ We are currently examining the role of beta-parvin, which competes with PARVA for ILK binding $^{36}$ and promotes Rac1 and Cdc42 activation and actin polymerization in response to integrin signaling by interacting with alpha-actinin ${ }^{37}$ and with alpha-PIX. ${ }^{38}$

In contrast to mild ILK-C expression, marked overexpression of ILK-C resulted in inhibition of hypertrophic signaling. It is currently unknown whether this effect is mediated by interference with prohypertrophic ILK-dependent signaling, such as those possibly mediated by beta-parvin, paxillin 
or other ILK-interacting proteins, or results from activation of the apoptotic program with caspase cleavage of prohypertrophic proteins. In mouse cardiac myocytes, deletion of ILK resulted in marked apoptosis without induction of hypertrophy. Based on our results, we hypothesize that while ILK and PARVA localization to costameres is essential for cardiac myocyte survival, ILK may act as a scaffold for the assembly of either pro- or antihypertrophic signaling complexes, including those involving alternative parvin and PINCH proteins, depending on extracellular stimuli and intracellular localization. Further study of ILK-dependent pathways is necessary to elucidate how the extracellular matrix, through integrin receptors, interacts with other prohypertrophic stimuli to regulate cardiac myocyte hypertrophy and survival.

\section{Acknowledgements}

This work was supported by the National Institutes of Health Grants HL068714-03 (to JS) and DK54639 (to CW).

\section{References}

1 Aikawa R, Nagai T, Kudoh S, et al. Integrins play a critical role in mechanical stress-induced p38 MAPK activation. Hypertension 2002;39:233-238.

2 Shyy JY, Chien S. Role of integrins in cellular responses to mechanical stress and adhesion. Curr Opin Cell Biol 1997;9:707-713.

3 Wei L, Wang L, Carson JA, et al. beta1 integrin and organized actin filaments facilitate cardiomyocytespecific RhoA-dependent activation of the skeletal alpha-actin promoter. FASEB J 2001;15:785-796.

4 Heidkamp MC, Bayer AL, Kalina JA, et al. GFP-FRNK disrupts focal adhesions and induces anoikis in neonatal rat ventricular myocytes. Circ Res 2002;90: 1282-1289.

5 Ross RS. The extracellular connections: the role of integrins in myocardial remodeling. J Card Fail 2002;8: S326-S331.

6 Valencik ML, Keller RS, Loftus JC, et al. A lethal perinatal cardiac phenotype resulting from altered integrin function in cardiomyocytes. J Card Fail 2002; 8:262-272.

7 Hannigan GE, Leung-Hagesteijn C, Fitz-Gibbon L, et al. Regulation of cell adhesion and anchorage-dependent growth by a new beta 1-integrin-linked protein kinase. Nature 1996;379:91-96.

8 Zhang Y, Chen K, Tu Y, et al. Assembly of the PINCHILK-CH-ILKBP complex precedes and is essential for localization of each component to cell-matrix adhesion sites. J Cell Sci 2002;115:4777-4786.

9 Fukuda T, Chen K, Shi X, et al. PINCH-1 is an obligate partner of integrin-linked kinase (ILK) functioning in cell shape modulation, motility, and survival. J Biol Chem 2003;278:51324-51333.

$10 \mathrm{Tu}$ Y, Huang Y, Zhang Y, et al. A new focal adhesion protein that interacts with integrin-linked kinase and regulates cell adhesion and spreading. J Cell Biol 2001;153:585-598.
11 Nikolopoulos SN, Turner CE. Integrin-linked kinase (ILK) binding to paxillin LD1 motif regulates ILK localization to focal adhesions. J Biol Chem 2001;276: 23499-23505.

$12 \mathrm{Wu}$ C, Dedhar S. Integrin-linked kinase (ILK) and its interactors: a new paradigm for the coupling of extracellular matrix to actin cytoskeleton and signaling complexes. J Cell Biol 2001;155:505-510.

13 Fukuda T, Guo L, Shi X, et al. CH-ILKBP regulates cell survival by facilitating the membrane translocation of protein kinase B/Akt. J Cell Biol 2003;160:1001-1008.

14 Attwell S, Mills J, Troussard A, et al. Integration of cell attachment, cytoskeletal localization, and signaling by integrin-linked kinase (ILK), CH-ILKBP, and the tumor suppressor PTEN. Mol Biol Cell 2003;14:4813-4825.

15 Delcommenne M, Tan C, Gray V, et al. Phosphoinositide-3-OH kinase-dependent regulation of glycogen synthase kinase 3 and protein kinase B/AKT by the integrin-linked kinase. Proc Natl Acad Sci USA 1998; 95:11211-11216.

16 Tu Y, Li F, Wu C. Nck-2, a novel Src homology2/3containing adaptor protein that interacts with the LIMonly protein PINCH and components of growth factor receptor kinase-signaling pathways. Mol Biol Cell 1998; 9:3367-3382.

17 Zhang Y, Guo L, Chen K, et al. A critical role of the PINCH-integrin-linked kinase interaction in the regulation of cell shape change and migration. J Biol Chem 2002;277:318-326.

18 Guo L, Wu C. Regulation of fibronectin matrix deposition and cell proliferation by the PINCH-ILKCH-ILKBP complex. FASEB J 2002;16:1298-1300.

19 Sun P, Wang XQ, Lopatka K, et al. Ganglioside loss promotes survival primarily by activating integrinlinked kinase/Akt without phosphoinositide $3-\mathrm{OH}$ kinase signaling. J Invest Dermatol 2002;119:107-117.

20 Wang XQ, Sun P, Paller AS. Inhibition of integrinlinked kinase/protein kinase B/Akt signaling: mechanism for ganglioside-induced apoptosis. J Biol Chem 2001;276:44504-44511.

21 Attwell S, Roskelley C, Dedhar S. The integrin-linked kinase (ILK) suppresses anoikis. Oncogene 2000;19: 3811-3815.

22 Persad S, Attwell S, Gray V, et al. Inhibition of integrin-linked kinase (ILK) suppresses activation of protein kinase B/Akt and induces cell cycle arrest and apoptosis of PTEN-mutant prostate cancer cells. Proc Natl Acad Sci USA 2000;97:3207-3212.

23 Yoganathan N, Yee A, Zhang Z, et al. Integrin-linked kinase, a promising cancer therapeutic target: biochemical and biological properties. Pharmacol Ther 2002;93:233-242.

24 Laser M, Willey CD, Jiang W, et al. Integrin activation and focal complex formation in cardiac hypertrophy. J Biol Chem 2000;275:35624-35630.

25 Johnatty SE, Dyck JR, Michael LH, et al. Identification of genes regulated during mechanical load-induced cardiac hypertrophy. J Mol Cell Cardiol 2000;32: 805-815.

26 Chen H, Huang XN, Stewart AF, et al. Gene expression changes associated with fibronectin-induced cardiac myocyte hypertrophy. Physiol Genomics 2004;18: 273-283.

27 Ogawa E, Saito Y, Harada M, et al. Outside-in signalling of fibronectin stimulates cardiomyocyte hypertrophy in cultured neonatal rat ventricular myocytes. J Mol Cell Cardiol 2000;32:765-776. 
28 Taylor JM, Rovin JD, Parsons JT. A role for focal adhesion kinase in phenylephrine-induced hypertrophy of rat ventricular cardiomyocytes. J Biol Chem 2000;275:19250-19257.

29 Ross RS, Pham C, Shai SY, et al. Beta1 integrins participate in the hypertrophic response of rat ventricular myocytes. Circ Res 1998;82:1160-1172.

30 Deng XF, Rokosh DG, Simpson PC. Autonomous and growth factor-induced hypertrophy in cultured neonatal mouse cardiac myocytes : comparison with rat. Circ Res 2000;87:781-788.

31 Terpstra L, Prud'homme J, Arabian A, et al. Reduced chondrocyte proliferation and chondrodysplasia in mice lacking the integrin-linked kinase in chondrocytes. J Cell Biol 2003;162:139-148.

32 Kim DJ, Park SH, Lim CS, et al. Cellular localization of integrin isoforms in phenylephrine-induced hypertrophic cardiac myocytes. Cell Biochem Funct 2003; 21:41-48.

33 Pham CG, Harpf AE, Keller RS, et al. Striated musclespecific beta(1D)-integrin and FAK are involved in cardiac myocyte hypertrophic response pathway.
Am J Physiol Heart Circ Physiol 2000;279:H2916H2926.

34 Yue Y, Lypowy J, Hedhli N, et al. Ras GTPaseactivating protein binds to Akt and is required for its activation. J Biol Chem 2004;279:12883-12889.

35 Yamaji S, Suzuki A, Sugiyama Y, et al. A novel integrin-linked kinase-binding protein, affixin, is involved in the early stage of cell-substrate interaction. J Cell Biol 2001;153:1251-1264.

36 Zhang Y, Chen K, Tu Y, et al. Distinct roles of two structurally closely related focal adhesion proteins, alpha- and beta-parvins, in regulation of cell morphology and survival. J Biol Chem 2004;279:41695-41705.

37 Yamaji S, Suzuki A, Kanamori H, et al. Affixin interacts with alpha-actinin and mediates integrin signaling for reorganization of F-actin induced by initial cell-substrate interaction. J Cell Biol 2004;165: 539-551.

38 Filipenko NR, Attwell S, Roskelley C, et al. Integrinlinked kinase activity regulates Rac- and Cdc42mediated actin cytoskeleton reorganization via alphaPIX. Oncogene 2005; 9 May [Epub ahead of print]. 